\title{
Le flux des textes français en Espagne : de Le beau Solignac (1880) de Jules Claretie à La ducha de Mariano Pina (1884)
}

\author{
Martinez-Rodriguez, Carlos
}

Universitat de València, Charles.Martinez@uv.es

\begin{abstract}
Resumen
La obra de Mariano Pina Domínguez, traductor y adaptador de obras francesas a la escena española. El objetivo de este estudio es analizar a este autor español, adaptador de obras francesas de Scribe, de Labiche, de Pailleron, de Bisson y de otros muchos, lo que permitirá descubrir textos que son el fiel reflejo de la sociedad francesa en la realidad española de la época. ¿ Mediante qué extraña alquimia Le beau Solignac (1880) de Jules Claretie se convierte en metáfora acuática en La ducha (1884) de Mariano Pina ? El estudio de dicha obra nos permitirá considerar las diferencias inherentes al mecanismo de transposición teatral de un idioma al otro. Con ese objetivo, observaremos que el ejercicio de reescritura será el resultado del público y de la sala en la que la obra va a ser interpretada. En último lugar analizaremos la metaforización del agua presente en las dos obras.
\end{abstract}

Palabras clave : Traducción ; teatro ; francés ; España ; siglo XIX.

\section{Résumé}

L'œuvre de Mariano Pina Domínguez, traducteur et adaptateur des lettres françaises à la scène espagnole. Le but de cette étude est d'analyser cet auteur espagnol, adaptateur des pièces françaises de Scribe à Labiche, en passant par Pailleron, Bisson et bien d'autres, ce qui permettra la découverte de textes qui sont le reflet fidèle de la société française dans la réalité espagnole. Par quelle étrange alchimie Le beau Solignac (1880) de Jules Claretie devient métaphore aquatique dans La Ducha (1884) de Mariano Pina? L'étude de cette œuvre nous permettra d'aborder les différences inhérentes au mécanisme de transposition théâtrale d'une langue à l'autre. Dans ce but, nous observerons que l'exercice de réécriture sera le résultat du public et de la salle où la pièce devra être jouée. En dernier lieu nous approfondirons dans la métaphorisation de l'eau présente dans les deux pièces.

Mots-clés : Traduction ; théâtre ; français ; Espagne ; XIXe siècle.

\begin{abstract}
The work of Mariano Pina Dominguez, translator and adaptor of several French pieces to the Spanish stage. The aim of this study is to analyse this Spanish author's work. He adapted Scribe's, Labiche's, Pailleron's and many others' work. Those versions helped his Spanish peers discover many texts that were a true reflection of the French society. How was the process of conversion of Le Beau Solignac (1880) by Jules Claretie into the aquatic metaphorical comedy called La ducha (1884) by Mariano Pina ? The analysis of this work will enable us to discover the inherent differences to the theatetrical mechanism of translation from a language to another one. With this aim in mind, we will realize that an adaptation turns out to be the result of the audience's preferences and the place where the play is performed. At last, we will focus on the metaphorical presence of the water in both plays.
\end{abstract}

Keywords : Translation ; theatre ; French ; Spain ; XIX century. 
Le beau Solignac est un roman d'aventures avec tous les ingrédients dont ce genre nécessite : la quête de l'amour, l’amitié, la solidarité, le patriotisme, le désir de richesse, la hiérarchie sociale et les topos habituels du genre.

Riche en péripéties, avec un rythme qui démarre dans le calme presque morne du bureau de la Préfecture à Paris, augmente sa tension dramatique à travers les pages jusqu'à atteindre son climax au moment de l'évasion de la prison du Temple, autrefois Tour du Temple, de l'un des protagonistes. Le roman abonde en personnages simples, avec une apparence très physique qui justifie de leur fonction dans l'histoire, sans entrer dans leur profondeur psychologique, et qui sont les archétypes d’une masse populaire qui ne cherche dans ces lectures qu'une brève fuite de la réalité.

Ce roman de près de quatre-cents pages se divise en deux parties Andréina et Louise de Farges, les deux grandes héroïnes de l'histoire. C'est dans la première partie que Mariano Pina y trouve son inspiration pour créer sa pièce $L a$ ducha.

\section{L'adaptation}

Mais comment parvient-il à cette métamorphose ? Le contexte historique disparaît. Les batailles napoléoniennes et les intrigues de la Cour sous le Premier Empire n'intéressent guère le public espagnol, qui garde encore le souvenir de l'invasion bonapartiste. L'histoire de Pina n'aurait que faire d'un arrière fond historique.

Ce qui intéresse le dramaturge espagnol, prolixe dans la composition de pièces légères, sans intention psychologique, mais riches en blagues, jeux de mots simples et sous-entendus qui créent des situations drôles, c'est plutôt les liaisons qui unissent les personnages, le complexe réseau sentimental qui se tisse entre eux et qui fait à ses yeux l'intrigue parfaite du vaudeville, dont il est maître.

Dans l'œuvre de Claretie, trois femmes sont l'essence du roman. Thérèse, Andréina, Comtesse d’Olona et la Comtesse Louise de Farges. Trois hommes jeunes sont la proie de leur désir. Le commandant Rivière, le colonel Solignac et le Comte d’Olona.

Thérèse est la jeune épouse du Commandant Rivière, Andréina, Comtesse d’Olona, séduit le jeune et beau Solignac, puis la Comtesse Louise de Farges, qui entre en scène à la fin de la première partie, pour essayer de séduire, elle aussi, le Colonel Solignac, sans se douter que quelqu'un d'autre cherche à la séduire, elle-même, dans le seul but de s'emparer de sa fortune. Il s’agit du Comte d’Olona, aristocrate italien complètement désargenté.

Pina Dominguez rescape l'essence de ces personnages et en crée des propres. Ainsi, il ne conserve que le prénom de Thérèse, qui est en son origine une femme perfide, voire libertine, qui trompe son époux avec un autre qui termine, faute de revenus, par l'abandonner pour courir d'autres fortunes (c'est bien le Comte d'Olona dont les billets d'amour à cette femme mariée, sont le détonnant de toute l'intrigue). La Teresa de La ducha, elle, est une femme simple, toute faite de bonté.

Si Thérèse, sachant son mari arrêté par la police, quitte le foyer familial pour s'enfuir avec son amant sans laisser de trace, Teresa, elle, refuse de quitter son mari rien qu'un seul mois, bien que ce soit pour répondre aux exigences d'un oncle qui garantit la fortune. Teresa poussera son mari à monter toute une farce, qui lui permette d'être près de lui tout le temps qu'il devra accompagner son oncle et sa cousine.

Thérèse ne pense qu’à elle, à ses sentiments, à sa honte après subir le dépit de son amant. Teresa aidera ceux qui l'entourent à trouver leur bonheur sentimental. Teresa aime, veut être aimée, et que les autres autour d'elle, s'aiment. C'est une femme simple, archétype des personnages de Pina.

Andréina, est une très jeune et très belle femme qui sous son apparence de femme mondaine, cache une fille fragile. Sa version espagnole s'appelle Clara, elle aussi jolie et jeune, mais en revanche d'une naïveté et d'une douceur sans revers.

Andréina aime le beau Solignac, elle guette sa proie, l’observe, lance sa ligne et attend que le colonel morde l'hameçon, ce qui ne se fait pas attendre. Elle sent son trophée en danger lorsqu’apparaît la comtesse de Farges.

Telle est la candeur de Clara, qu’elle n’ose avouer qu’à la dernière scène de la pièce qu'elle aime Narciso.

Quant aux hommes, Claretie joue avec l'espèce la plus estimée des romans d'aventures dix-neuvièmistes : les militaires. 
Aussi bien les femmes que l'empereur lui-même appellent le colonel Solignac, le beau Solignac. C'est l'essence même du courage, du patriotisme, de l'amitié, de la camaraderie, de l'élégance. Il ferait tout et n’importe quoi pour celui qu’il appelle son frère d’armes : le commandant Rivière. À tel point qu'il l'aide à s’évader de la prison la plus hermétique qui soit à cette époque à Paris, la Tour du Temple, tout en risquant et sa réputation, et sa carrière, et sa vie. Mais Solignac n'est pas, dans son apparence martiale, impassible. Il succombe aux charmes d’Andréina, puis se laisse gâter par Louise de Farges.

Jacinto est en quelque sorte le beau Solignac espagnol. Il n'est ni militaire, ni aussi beau que le colonel parisien. Il s'agit d'un simple fonctionnaire intérimaire qui attend éternellement qu'un poste se libère dans un ministère pour accomplir son rêve de travailler. D’abord mû par l’intérêt économique, puis influencé par les sentiments d’amour de ceux qui l'entourent, il acceptera de jouer un rôle protagoniste dans la farce de Pina. S’il hérite une seule caractéristique du personnage français, c’est bien le sens de l’amitié, le dévouement qu’il sent pour Enrique. De là qu’il se prête au jeu qu'on lui propose.

Mais, qui est-ce Enrique ? Il s’agit du héros de La ducha. C'est un homme simple, qui aime une femme simple qui n’a pas de dote. C’est la bonté par nature. Il aime son vieil oncle et ne veut pas le décevoir, mais il ne peut pas satisfaire son dessein de se marier avec sa propre cousine, non seulement parce qu’il est déjà marié en secret avec celle qui lui a volé le cœur, mais surtout, parce qu'il ne sent rien pour sa cousine. Enrique est un homme digne, et c'est cette dignité qui le rapproche du Commandant Rivière.

Ce dernier est un époux aimant, trompé et trahi, qui a un tel sens de la dignité qu’il pardonne à sa femme, mais profondément blessé et la sachant poursuivie par la loi, ne la veut plus auprès de lui et demande à son frère d'armes, qu'il s'occupe de la cacher.

Dans une autre échelle d’intensité, puisque les cadres de l'action sont très différents, Jacinto est pour Enrique, ce que le beau Solignac est pour le Commandant Rivière. Si Solignac délivre Rivière de son emprisonnement, Jacinto se prête au jeu des mensonges pour aider Enrique.

Voici donc le cadre et les personnages, mais qu'en est-il de l'intrigue ? Le beau Solignac est aussi un roman sentimental, une histoire d'amour, de séduction, de trahison, et de dépit amoureux. Les uns aiment les autres qui ne se sentent pas correspondus et des tierces viennent s'y mêler pour compliquer les affaires du cœur, qui sont loin d'être simples par elles-mêmes. Et c'est de là, principalement, que Mariano Pina Dominguez s’inspire du beau Solignac et tire toute la moelle de sa pièce.

Enrique s'est marié en secret avec Teresa. L'oncle Gregorio veut caser sa nièce Clara, et vise pour ce faire, son neveu, Enrique. Clara n'aime pas Enrique et est amoureuse du secrétaire de son vieil oncle, le jeune Narciso, qui lui, est épris de la jeune fille, sans oser l'avouer à son patron.

L'action déborde comique de situations, de jeux de scène, de rebondissements qui n’ont pour but que de captiver l'attention du spectateur sans repos, à travers le rire, et l’attente que la vérité que cache la farce se dévoile enfin.

Ce vaudeville présente de fausses relations sentimentales qui sont censées aboutir à un dénouement contraire à celui que l’intrigue par elle-même semble tisser.

Ainsi donc, Gregorio qui voulait marier sa nièce à son neveu et son secrétaire à une autre, termine par accepter sans le savoir, la liaison de son employé avec sa nièce et de renoncer à l'accord établi avec son neveu, de sorte à ce que ce dernier puisse aimer librement, celle qui en réalité est déjà son épouse.

\section{Les métaphores aquatiques dans Le beau Solignac}

L'eau est une constante dans l'œuvre de Claretie, et le plus frappant est que Pina Dominguez respecte cette omniprésence qu'il transpose dans sa pièce.

Ainsi, des deux héros de Claretie, celui qui débute l'action, cet homme intègre qui refuse de trahir ses compagnons d'armes, malgré avoir été trahi lui-même par sa femme, ce colonel qui pouvant vendre le nom de celui qui a outragé sa femme, ne le fait pas, cet homme ferme et digne en toute circonstance, se nomme Rivière. Et comme l'eau qui coule 
paisiblement, lorsqu'il rencontre sa femme après que son amant l'ait abandonnée, au lieu de lui faire une scène de dépit amoureux, voire même un scandale d’homme déshonoré, calmement il la pardonne, paisiblement il la protège.

L'histoire ayant lieu sous Napoléon, c’est le moment où la France a connu bien de victoires, « des victoires où le sang français coule à flots » (Claretie, $1880: 11$ ), comme si les français offraient à leur empereur leur propre sang pour nourrir le champ de bataille, pour allaiter le champ de la gloire. Mais si « on le versait [autrefois] pour l'indépendance du pays, on le répand aujourd'hui dans des guerres de conquêtes pour l'asservissement du monde.» (Claretie, 1880 : 11). Et le verbe verser exprime combien les artères des soldats se vident de ce liquide précieux à la vie, combien ces combattants exsangues irriguent de mort la terre, la France.

Le sang est un liquide mortel lorsqu’on le perd, mais l'eau est un liquide vital, lorsque les émotions font sécher notre bouche, lorsque la salive de l'homme qui pressent le pire manque, et qu'il n'y a plus rien à avaler. C'est alors que face à la réaction silencieuse de Claude, on entend : «De l'eau, dit Fouché, sans nulle émotion » (Claretie, 1880 : 14). Et Claude Rivière, que l'émotion du mauvais présage avait séché la bouche, répond : «Merci... rien... merci » (Claretie, $1880: 14)$.

Changeons de contexte, allons chercher un des personnages secondaires de cette passionnante aventure, Mademoiselle de la Rigaudie, vieille fille riche, fille du dernier marquis de la Rigaudie et grande amie du beau Solignac. Elle décide d'aider à l'évasion du commandant Rivière. Elle habite un hôtel particulier situé non loin de la prison du Temple, et reçoit ses amis intimes dans «son vaste salon aux panneaux décorés de marines de Joseph Vernet » (Claretie, 1880 : 66), à qui Louis XIV commanda une collection de tableaux des ports de France. Ces tableaux sont considérés de véritables témoignages de la vie dans les ports français de l'époque et font de Vernet un des plus grands peintres de la Marine française.

Un des traits caractéristiques de ce roman d'aventures est la richesse de contrastes. De la mer majestueuse de l'œuvre d'un peintre sublime, nous passons à l'eau sous forme de vapeur malodorante qui imprègne les planches de bois qui forment la porte du cachot de Rivière, ainsi donc: «es ais en étaient épais sans doute, mais l’humidité les avait disjoints » (Claretie, $1880: 74$ ).

Un des instants les plus poétiques de l'œuvre est certes l'entrée sur scène d'Adréina, marquise d’Olona, qui arrive en pleine revue militaire, un des grands divertissements des Parisiens de cette époque. En plein défilé son landau s'ouvre chemin entre les gens, « [entre d'autres] calèches aux panneaux gros bleu, vert d'eau, cerise, ou vert olive, ne pouvant circuler dans ce fleuve humain, demeurant immobiles des deux côtés de la rue, et la foule roulait, si l'on peut dire, entre ces deux rives d'équipages » (Claretie, 1880 : 79). Andréina se tient debout, c’est une femme d’une vingtaine d’années, admirablement belle, avec «[les] yeux verts comme l'eau profonde » (Claretie, $1880: 80$ ), et devant elle, «le flot s’était d'ailleurs ouvert » (Claretie, $1880: 80$ ) pour la laisser passer.

C'est pendant la revue qu'elle connaît le beau Solignac, et qu'elle lui offre un bouquet de roses que celui-ci mettra dans un vase plein de la potion qui rend la beauté des roses éternelle : « Il avait, ce bouquet partagé en deux, laissant la moitié des fleurs à l’hôtel, les tiges baignées dans une eau pure » (Claretie, 1880 : 87).

Mais non satisfaite de son premier élan, elle donnera rendez-vous au beau militaire en plein centre de Paris, d'un Paris qui grouille de vie, d'endroits à la mode. La première image aquatique dans ce cas-là est celle de la société parisienne comparée à la cour de la souveraine d'une île mythique : « Toutes les divinités de l’Olympe [...] Comus coudoyant Mercure, Plutus souriant à la reine de Cythère » (Claretie, 1880 : 86) puis, après la suite royale, vient l'effervescence d'une ville toujours bouillonnante d'animation. L'activité de la ville ne tarde pas à se montrer riche en symboles qui rappellent l'eau : à travers les cafés en tant que nom de lieux, en tant que boisson à la mode, résultat de l'infusion dans l'eau bouillante des graines du caféier, en citant les différentes manières de servir cette boisson selon la spécialité de chaque restaurant, de chaque terrasse, en parlant des différentes variétés de la graine telles que le moka, puis en évoquant les personnalités de l'époque telles que la Belle Limonadière, célèbre pour sa beauté et pour la boisson qu’elle servait : de l'eau fraîche avec du jus de citron et quelques cuillerées de sucre, soit de la limonade, boisson désaltérante. Ainsi donc :

Le café de Foy, dont les glaces étaient célèbres; le café de la Rotonde ; le café des mille Colonnes, où la Belle Limonadière [...] [étonnait] les étrangers ; le café Sabbatino, où les officiers et les élégantes allaient consommer le 
punch à la romaine, aussi célèbre que le moka du café Lemblin ; le café des Chartres, [...] le café de Valois ; [...] le café des Étrangers ; [...] le café Montausier [...] où se réunissaient les gens du bel air [...] et qui ne dînaient que chez Véry. (Claretie, $1880: 86$ )

Mais l'eau a aussi une face sombre, elle est aussi le symbole du malheur, de la tristesse. Lorsque Claude Rivière s'évade, la vapeur est la protagoniste du décor, des passages sombres entre la prison du Temple et l’hôtel de la Rigaudie. Le chemin rustre et caillouteux, l’humidité souterraine abondante font que l'évasion soit pénible et douloureuse : «Il se hâta [...] trébuchant, se retenant aux murs humides, les épaules glacées par des gouttelettes qui suintaient comme des larmes coulent [...]» (Claretie, 1880:101). L’humidité devient la fidèle compagne du commandant tout le long du chemin : «C'était les suintements des voûtes qui tombaient en gouttelettes dans les flaques stagnantes ». (Claretie, 1880 : 101). Mais les instants d’inquiétude passent, Claude réussit à se sauver et la nuit l'attend. Là, l’humidité prend une autre tournure, rafraîchissante, apaisante : « Avec le serein qui tombe » (Claretie, 1880 : 103) et il faut se protéger.

Et si la nuit est devenue accueillante, la situation le devient aussi, la cellule reste loin maintenant, et devant lui, ceux qui l'aiment, son père et son ami. Les fluides prennent alors une forme libératrice des émotions jusque-là retenues : « Et le commandant Rivière sentait sur son front chargé de soucis, tomber ces chères larmes paternelles qui lui semblaient si douces et bonnes ». (Claretie, $1880: 104)$.

Dans un autre univers, Andréina se dépouille de l'un de ses plus précieux bijoux et l'offre à son frère pour le sortir de sa misère. La transparence de l'eau pure renvoie les rayons de toute source lumineuse. C'est ici la métaphore idéale pour nuancer la perfection du joyau : «Elle [...] détacha de ses oreilles deux diamants qui étincelaient comme deux gouttes d'eau sur des feuilles de roses ». (Claretie, $1880: 119$ )

Le liquide cristallin protège aussi le commandant Rivière qui ne peut pas vivre librement, car il est tout de même un fugitif de la justice et trouve refuge « dans une petite maison [...] de la Rue du Château-d’Eau ». (Claretie, 1880 : 124)

L’eau se présente aussi comme élément rédempteur de la souffrance humaine. Thérèse Rivière, abandonnée par son amant et soumise dans un profond désarroi, ne songe qu’à se donner la mort, soit par l'ingestion d'une boisson venimeuse : «Si Agostino [avait] laissé à la serrure la clef du laboratoire [...] [elle] aurait bu, au hasard, quelque poison » (Claretie, 1880 : 138) soit en se laissant elle-même avaler par les eaux profondes de la Seine : " N'avait-elle point le fleuve, le grand fleuve qui, là-bas, a tant de fois roulé et étouffé dans ses eaux les douleurs humaines ? » (Claretie, 1880 : 138). Et Thérèse ne sait plus attendre. Devant la honte de pouvoir rencontrer celui qu'elle a trahi, elle est résolue à se livrer aux profondeurs du fleuve : «Elle voulait attendre qu'il fît tout à fait nuit pour descendre sur la berge et aller vers la mort lentement, en avançant pas à pas, jusqu'à ce que l'eau dépassât son visage, ou pour se précipiter follement du haut d'un pont » (Claretie, 1880 : 138). Mais elle revient sur ces pas, et elle préfère se donner au châtiment de la souffrance qui fait pleurer : «j' [ai] à expier ce que j' [ai] fait autrement que par la mort, par une vie de souffrances et de larmes » (Claretie, 1880 : 142). L’effet de l'eau sur les vêtements de Thérèse la rend piteuse : «Quels vêtements, vous êtes toute traversé ! [...] Elle prenait entre ses mains la jupe mouillée de Thérèse et, la tordant, elle la montrait ruisselante » (Claretie, 1880 : 143). Puis les larmes de souffrance deviennent des larmes de honte : « Thérèse sentit ses yeux s'emplir de larmes et sa poitrine oppressée se souleva gonflée par un violent sanglot » (Claretie, 1880 : 147).

En conclusion et pour clore ce parcours sur les symboles aquatiques de Le beau Solignac, il faut faire référence à la rencontre de la deuxième héroïne de cette histoire, la comtesse de Farges, qui rencontre pour la première fois Solignac dans les jardins du Ministère lors d'une réception. Dans ce cadre, l'eau prend un tout autre accent, lyrique, romantique cette fois : « C'est la caresse du vent sur l'eau limpide, fraîche, attirante, d'un lac sans tache » (Claretie, 1880 : 163). Et les eaux sont peuplées de personnages mythologiques qui accompagnent les amoureux pendant leur flânerie : « [...] une statue de l'Amour [...] faisant, de l'index de la main droite, des signes de menace à des nymphes placées, ça et là, sur un piédestal autour du rond-point [...] comme la Galathée du poète latin » (Claretie, 1880 : 163). « Devant le piédestal de chacune de ces nymphes, un banc de bois attendait les promeneurs. » (Claretie, 1880 : 163). 


\section{Les symboles hydriques dans La ducha}

Dans l'œuvre de Pina Dominguez, l'eau y est présente dès le titre : La ducha, bien qu'elle se fasse remarquer tout autrement que dans l'œuvre romanesque de Claretie. Les références sont moins éparses, peut-être parce que moins implicites, bien plus directes, bien plus évidentes. Le texte espagnol n’est pas descriptif, il s'agit d'un vaudeville où l'essentiel est l'action, le comique de situation. Le style n'a point la même importance que dans le texte français.

Ainsi donc, juste deux brèves allusions à l'eau dans l'acte I. La première à la scène V, lorsque Gregorio, l'oncle d'Enrique, lui parle de son intention de partir un mois en vacances dans un balnéaire, comme il était à l'usage pour la bourgeoisie européenne au XIXème siècle et au début du XXème. L'eau est ici, médicinale, curative, et possède des qualités bienfaitrices dont on ne saurait se passer : «Gregorio : Escucha. Todo está pensado. Mi sobrina y yo partimos para los baños. En esta época todo el mundo necesita tomar aguas. » (Pina, 1884 : 12).

Deuxième allusion, à la scène XV qui vient clore le premier acte, l'eau devient alors le stratagème qui permettra aux jeunes Enrique et Teresa de se rencontrer à l'insu de l'oncle Gregorio : «Enrique : y mientras [el tío] toma sus duchas tranquilamente... » (Pina, 1884 : 25). «Jacinto : Sus duchas ? La verdadera ducha para el tío ya sé yo cual es. [...] ducha de impresión vertiginosa » (Pina, 1884 : 26) lorsqu’il découvrira le mariage secret de son neveu.

Dans l'acte II, les séances curatives commencent et l'élément hydrique commence ici à faire ses effets, les personnages se détendent. Ils vont à la source : « Narciso : Clarita, había olvidado usted su abrigo en el manantial [...] Gregorio : [...] [aquí estamos] bebiendo agua sulfurosa” (Pina, 1884 : 31). L’acte se déroule entre activités diverses qui tournent autour de la consommation d'eau : « Teresa à Clarita : Tomaremos juntas las aguas » (Pina, 1884 : 35). Les verbes se rapportent aux activités physiologiques liées à l’eau : « Médico : le costará a usted trabajo la deglución ? [...] Jacinto : hace ya muchos años que apenas trago. » (Pina, 1884 : 37). Puis les effets bienfaiteurs se manifestent : " Gregorio : (cogiendo el brazo de Narciso) parece mentira como desarrollan el vigor estas aguas !... . » (Pina, 1884 : 37 ). Et il ajoute quelques répliques après : « parece mentira cómo animan éstas aguas. » (Pina, 1884 : 39).

L’eau est à tel point curative que certains viennent y passer de longs séjours : « Teresa : y hace mucho que están ustedes en los baños ? » (Pina, $1884: 41)$.

Toutes les manières de consommer les eaux se pratiquent au balnéaire, et le comique de situation est servi :

Jacinto : [...] a las ocho, baño frío de treinta minutos. Pulverización directa. Seis cortadillos de la fuente de Minguirriturri y tres duchas de chorro. Gregorio : Hombre, lo mismo que yo. Jacinto: Ah! Toma usted todo esto ? Gregorio : Todo. Jacinto : Y no ha reventado usted todavía ? Gregorio : Reventar? ¡Al contrario! Pues si tengo un apetito atroz. Jacinto : Esto abre el apetito ? Gregorio : Ya lo creo. Jacinto : Voy a tomar una ducha! (Pina, $1884: 44)$.

Et par moments ses activités servent de piège pour tromper le vieil oncle qui pense, en tournure de vaudeville, que c'est justement un autre qui est trompé : «Gregorio : y mientras toma la ducha, se la pegan como a un chino. » (Pina, 1884 : 48).

Mais l'eau peut être aussi un calvaire, et tandis que les amoureux essaient de préparer l'oncle pour lui expliquer la vérité, le pauvre Jacinto qui par amitié se prête à tout, subit son calvaire :

Enrique : [...] de dónde viene usted ? Jacinto : de tomar la primera ducha. Yo creo que no resisto la cuarta. [...] Enrique : aquí va usted a engordar de lo lindo. Jacinto : ¡ Pues ya lo creo ! Sabe usted lo único que siento ? Enrique : El qué ? Jacinto : El agua. Enrique : que agua ? Jacinto : el agua Minguirriturri. Me han hecho beber seis vasos y ni los mismos demonios lo podrían pasar, es tinta pura, don Enrique. Enrique : [es que Jacinto] es un héroe! Jacinto : no, no tanto. Enrique : Sí señor, [soporta] las duchas, los baños, todo por nuestra causa. (Pina, 1884 : 49).

L'acte III est l'acte de clôture et par conséquent le moment où l'eau devient la grande vedette. A commencer par le lieu, la salle d'eaux du balnéaire, et tous les éléments reliés à son usage. La didascalie initiale est précise : 
La sala de pulverizaciones. Vénse los aparatos colocados en la pared. Contra el testero del foro, un armario lleno de toallas, paños, baberos de hule, etc. [...] banquetas en la escena. Perchas donde los bañistas cuelgan sus abrigos y todos los detalles propios de estos lugares. [...] D. Jacinto, el Pollo, el señor Gordo y la primera bañista toman la pulverización, cada cual en su aparato. [...] Los demás hacen gárgaras, ó se preparan para las pulverizaciones. Las camareras preparan las pulverizaciones a unos y a otros, doblan las tollas [...]. (Pina, 1884 : 53).

Tout au long de cette scène, les verbes marquent l’usage de l'élément aquatique : « Mamá : Pulverízate pronto y abre bien la boca [para que entre bien el chorro] [...] Usted para qué las toma ? Jacinto : Yo ? Para que no se diga!... [...] Mamá : [...] desde que llegó usted a los baños se está usted hinchando. Jacinto : (Ya lo creo! de tanto tragar!) (Pina, $1884: 54)$.

Les blagues légères tournent autour du précieux fluide : «Jacinto : [...] la moda exige que se ponga uno enfermo todos los veranos [y deba venir a tomar las aguas]. Mamá : Usted sin embargo tiene la carne fofa. Jacinto : ¿ cómo fofa? Mamá : [...] Sí, como si tuviese hidropesía » (Pina, 1884 : 54).

Certains verbes se répètent constamment, puisque les uns posent la même question aux autres : «Pollo : Voy a tomar mi baño, ¿̇ se ha bañado usted ya ? Jacinto : Todavía no. » (Pina, 1884 : 54).

Et les commentaires entre les malades sont tous liés aux effets positifs de l'eau, et aux troubles de santé produits par la non consommation de l'élixir miraculeux :

\begin{abstract}
Doctor : traga usted bien ? Jacinto : Yo mismo me asusto. Doctor : que nieguen luego la bondad de estas aguas. [...] Vea usted un enfermo que llega pálido, desfallecido, con la garganta ulcerada y el pulmón afecto [...] y a las veinticuatro horas lo tiene usted encarnado, sonriente y tragando sin dolor alguno, no es asombroso ? Jacinto : Oh! Asombrosísimo. [...] Doctor : Que aguas! Que aguas !... [...] Mañana una purga. La que usted quiera. [...] Cuatro duchas, la inhalación, la pulverización, los baños fríos, y no tenga usted miedo. Jacinto : Miedo ? No me conoce usted a mí ! Doctor : Le garantizo a usted la curación completa. Y estoy seguro que todos los años volverá usted por agradecimiento. (Pina, $1884: 54)$.
\end{abstract}

\title{
Conclusion
}

Et pour finir avec le texte, le leitmotiv que l’on ne peut jamais oublier : «Pero que aguas, que aguas ! Estas aguas son prodigiosas » (Pina, $1884: 54)$.

Et elles sont, en effet, si prodigieuses qu'elles font que le dénouement en fin de l'acte III soit, comme dans tout vaudeville, heureux. Les amoureux se rencontrent, le mensonge qui s'est bien compliqué tout au long des trois actes, se dévoile, et le comble du comique est que l'effet purificateur des eaux fait que l'oncle Gregorio qui voit tout son projet de mariage démonté, ne se fâche guère, mais se sent plutôt hilare, que son neveu et sa nièce aient trouvé leur bonheur sans son intervention. N'est-ce pas le vrai prodige de l'eau ?

\section{Références bibliographiques}

Claretie, Jules (1880). Le beau Solignac. Paris. Bibliothèque illustrée. Source : Bibliothèque Nationale de France, département Littérature et art, 4-Y2-627.

Pina Dominguez, Mariano (1884) La ducha. Juguete cómico en tres actos. Madrid. Sevilla 14, Principal. 